\title{
Risky Choices in Medical Decision Making: A Comment
}

\author{
by M. F. Drummond*
}

\section{Introduction}

Professor Weinstein has produced a stimulating paper which raises a wide range of conceptual, methodological and policy issues. Since my own interest is in the methods for assessing the costs, risks and benefits of health care programmes, and the way that the results of such analyses can be used to bring about a more efficient delivery of health care, I shall concentrate on the section of this paper dealing with choices under resource constraints. However, since Professor Weinstein's paper is itself so well integrated, I shall inevitably stray into other sections.

\section{The relevance of costs to health care decisions}

Professor Weinstein rightly emphasises the notion of opportunity cost and its relevance to choices under resource constraints, but suggests two reasons why physicians may not consider costs in making clinical decisions. Certainly it is relevant to distinguish between clinical decisions (made on behalf of the individual patients) and health care planning decisions (made of behalf of a group or community of patients). Whilst few would deny the importance of costs to the latter, many clinicians would argue that, for ethical reasons, costs should not enter into the former. There is obviously an inconsistency here, because as soon as resources are being involved, by definition the patient cannot be considered in isolation; more treatment for one individual must mean less for someone else (Drummond, 1980). The economist would therefore argue for a situation where clinicians were working to a protocol that was thoroughly researched in terms of cost-effectiveness (WHO, 1981) or were provided with a clinical budget (Wickings et al., 1983) that revealed the opportunity costs of the resources used in treatment.

However, one aspect of this which may have been overlooked is that the community may not be indifferent to the methods by which resources are allocated at the clinical level. That is, there may be a higher process utility associated with a situation where the clinician appears to be doing 'his best for the patient' and not working to an efficiency-determined protocol or

* Acting Director and Senior Lecturer in Economics Health Services Management Centre, University of Birmingham, United Kingdom 
facing up to resource constraints. It is, of course, an empirical matter whether members of the community, if asked, would be willing to accept the current level of inefficiency in clinical decision making for any associated higher quality in the doctor-patient relationship.

Sometimes the extra costs resulting from the undisciplined use of health services are quite great. For example, Levine et al. (1985) have recently estimated that in the diagnosis of cancer of unknown primary origin, a protocol of comprehensive diagnosis and treatment (searching for every possible primary site) would cost around $\$ 2$ million per annum more (for Ontario) than a limited protocol only investigating sites for which an effective systemic therapy were available. If, on being unable to find the primary site, all patients were given chemotherapy, even though its effectiveness were unproven, this would cost in a further $\$ 5,000,000$ more per annum than symptomatic care. (Under all options patients having cancers for which effective systematic therapy were available would received treatment.) Evidence of the effectiveness of therapy for other cancers is poor. Levine et al. estimated that a policy of comprehensive diagnosis and therapy would result in 10 extra patients knowing the primary site of the tumour and (optimistically) 5 extra patients being alive at the end of the first year, with a poor long-term prognosis. Such a policy would cost around $\$ 7,000,000$ per annum more than limited diagnosis and treatment.

Perhaps in such cases one solution would be for only the lower cost protocol to be funded by the third-party payer. Then if individuals, based on the information presented to them, wished to have additional procedures performed they should be free to do so.

\section{Methodological issues in assessing the costs and benefits of health care choices}

Professor Weinstein's paper raises many interesting points. I wish to highlight three which I consider particularly important. First, there is the issue of the quality of evidence typically assembled in studies of the cost-benefit type. Frequently, economists base their analysis on poor medical evidence and are consequently criticised. However, it is often the case that good clinical or epidemiological data do not exist and, since a decision on whether a new treatment or programme should be launched needs to be taken, the skilful use of sensitivity analysis can be helpful. However, all too frequently analysts ignore the shape of the probability distribution of key parameters, presenting only the results for their 'best guess' and high and low estimates.

In addition more effort should be made to undertake economic analysis alongside controlled clinical trials, from which evidence of the effectiveness of therapies is obtained. Drummond and Stoddart (1984) have reviewed the arguments for and against the inclusion of economic analysis in clinical trials, identified the main criteria for undertaking economic analysis, and suggested a policy for 'phasing in' the analysis so as to minimise wasted time and effort.

It is also worth noting that while the estimation of costs does not pose as many difficulties, there are often deficiences in the methods used in published papers. In particular, certain costs (notably those falling outside the health sector) are often omitted and average costs (especially the hospital per diem figure) are often used unthinkingly. Drummond et al. (1986) also note that in many published papers inadequate information on costing methodology is given and it is impossible to tell whether the methods used are satisfactory. 
Second, as Weinstein points out, the assessment of the utility of health outcomes is now an important feature of many economic evaluations in the health care field. There are broadly two approaches to utility assessment; direct measurement of the utilities of the relevant group (e.g. patients or members of the general public), or assessment by a consensus-forming exercise involving experts in the field, backed up by extensive sensitivity analysis. Thompson (1982) has recently criticised the measurement approach in terms of the validity and accuracy of the values obtained and suggested that on occasions it may be unethical. However, Torrance (1982), a proponent of the measurement approach, argues that criticisms of lack of validity and accuracy are unfounded, particularly in respect of the mean utility values obtained from a group of individuals. (It is accepted that individuals' utility values for a given health state vary quite widely.) Since for planning decisions it is the group values that are most relevant it would seem beneficial to proceed with these experiments and at the same time to explore how the utility values of various groups differ (Wolfson et al., 1982). If the differences are not great, or are systematic, it may be possible to avoid time consuming, and possibly distressful, utility assessments in many situations in the future.

Finally, Weinstein outlines in this paper and others (Weinstein and Stason, 1977) a formulation of the cost-effectiveness model. It should be noted that different authors use different formulations, and that two contentious issues are whether one excludes or includes (i) productivity gains or losses associated with treatment and (ii) costs of health services in later years of life. Weinstein excludes the former but includes the latter. Economists are divided on whether productivity gains/losses should be included. On the one hand they represent resource changes in the same way as the consumption or saving of health care costs. On the other hand their inclusion in the analysis biases health care decisions in favour of those who are economically active, descriminating against the elderly or unemployed. Most economists also have concerns about the way of measuring productivity changes (market wage rates) and have doubts about whether, given the current level of unemployment, in many countries, production is actually lost when illness prevents a given individual from working.

The inclusion of health care costs in added years of life also presents problems, particularly for prevention programmes, although if they are far into the future discounting to present values reduces their numerical impact on the analysis. Certainly these costs should be included if the quality or quantity of life-years gained as a result of treatments given in later life are included in the denominator of the cost-effectiveness model. On the other hand it seems unfair to penalise (say) a hypertension screening programme because individuals living to an older age may be given expensive, possibly ineffective, surgery and chemotherapy for their cancers. Surely these treatment decisions should be examined separately.

\section{Comparison of costs and benefits across a wide range of programmes}

Although economic evaluations can help us examine choices within a given health care field, there is also a need to assess priorities between (say) investments in renal disease and coronary heart disease. As Weinstein points out, there are now studies that compare options within health care in terms of their cost per life-year gained or cost per quality-adjusted lifeyear (QALY) gained (Kaplan and Bush, 1982). A recent example (Williams, 1985) is shown in Table 1. Weinstein also cites studies which compare investments in health care with those in highway safety, and environmental and occupational health (Graham and Vaupel, 1981). Whilst they are thought provoking, particularly for those policy-makers whose implicit values 
are being made explicit, such studies need to be treated with caution. There may be differences in study methodology, other benefits of options apart from life extension or improvement, and other factors pertinent to the choice (such as issues of distributive justice). There are also the wellknown factors affecting community perception of risk, e.g. whether the individuals at risk are identifiable, whether the risk is voluntary or non-voluntary, the nature of the risk and the unfamiliarity of the risk (Royal Society, 1983).

However, there remains the possibility that the price being paid to reduce risk in different situations varies because those taking decisions do not think through choices in a systematic way. This is what I believe to be the main contribution both of analyses of this type and Professor Weinstein's paper.

Table 1: Costs and consequences of investments in different medical treatments

Strong candidates for expansion

Pacemakers for heart block

Hip replacement

Valve replacement for aortic stenosis

CABG for severe angina with LMD

CABG for severe angina with $3 \mathrm{VD}$

CABG for moderate angina with LMD
Cost per QALY gained

$(1985, \mathfrak{f})$

\section{0}

750

950

1,040

1,270

1,330

\section{Less strong candidates}

CABG for severe angina with 2VD

$\mathrm{CABG}$ for moderate angina with $3 \mathrm{VD}$

PTCA for severe angina with IVD

CABG for mild angina with LMD

Kidney transplantation (Cadaver)

PTCA for moderate angina with IVD

Source: Williams (1985).

Note: $\quad$ QALY $=$ Quality-adjusted life-year.

$\mathrm{CABG}=$ Coronary artery bypass grafting.

PTCA = Percutaneous transluminal coronary angioplasty 


\section{REFERENCES}

1. DRUMMOND, M.F., [1980]: Principles of economic appraisal in health care. Oxford, Oxford Medical Publications.

2. DRUMMOND, M.F., and STODDART, G.L. [1984]: "Economic analysis and clinical trials". Controlled Clinical Trials; 5: 115-128.

2a. DRUMMOND, M.F., LUDBROOK, A., LOWSON, K.V., and STEELE, A. [1986]: Studies in economic appraisal in health care: volume two. Oxford, Oxford Medical Publications.

3. GRAHAM, J.D., and VAUPEL, J.W. [1981]: "Value of a life: what difference does it make?" Risk Analysis; 1(1): 89-95.

4. KAPLAN, R.M., and BUSH, J.W. [1982]: "Health related quality of life measurement for evaluation research and policy analysis". Health Psychology; 1(1): 61-80.

5. LEVINE, M., DRUMMOND, M.F., and LABELLE, R.J. [1985]: "Cost effectiveness in the diagnosis and treatment of carcinoma of unknown primary origin". Canadian Medical Association Journal; 133: 977-987.

6. ROYAL SOCIETY [1983]: Risk assessment. Report of a Royal Society Study Group. London, The Royal Society.

7. THOMPSON, M.S., and COHEN, A.B. [1982]: "Should we measure personal valuations of perinatal outcomes?" In J.C. Sinclair (ed). Clinical and economic evaluation of perinatal programs. Vail, Colorado, Mead Johnson Symposium on Perinatal and Development al Medicine No. 20.

8. TORRANCE, G.W. [1982]: "Preferences for health states: a review of measurement methods". In J.C. Sinclair (ed) op cit).

9. WEINSTEIN, M.C., and STASON, W.B. [1977]: "Foundations of cost-effectiveness analysis for health and medical practices". New England Journal of Medicine; 296: 716-721.

10. WICKINGS, H.I. et al. [1983]: "Review of clinical budgeting and costing experiments". British Medical Journal; 286: 575-569.

11. WILLIAMS, A. [1985]: "Economics of coronary artery bypass grafting". British Medical Journal; 291: 326-329.

12. WOLFSON, A.D., SINCLAIR, A.J., BOMBARDIER, C., and McGEER, A. [1982]: "Preference measurement for functional status in stroke patients; inter-rater and inter-technique comparisons". In R. Kane and R. Kane (eds). Values and long-term care. Lexington (Mass.), DC Heath and Co.

13. WORLD HEALTH ORGANIZATION (Regional Office for Europe) [1981]: Guidelines for health care practice in relation to cost-effectiveness. Euro reports and studies 53. Copenhagen, WHO. 